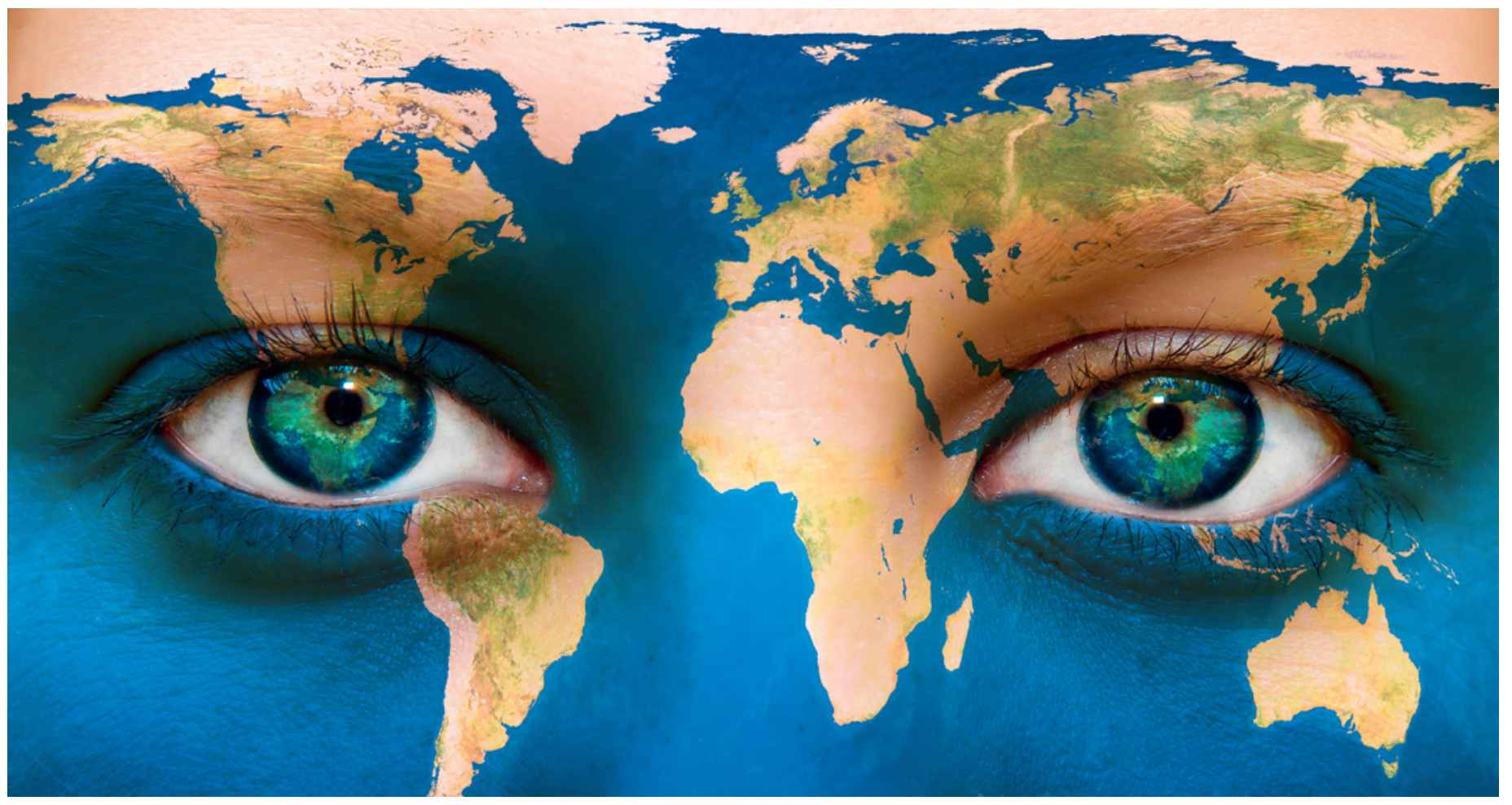

\title{
Dialogue intergénérationnel
}

\section{Jean Martin}

Dr méd., membre de la rédaction

Devant les défis lancés quant à l'avenir des générations futures, il est essentiel de promouvoir des échanges entre jeunes et moins jeunes - ou plus du tout jeunes -, d'avoir un dialogue adéquatement informé. L'association «Grands-parents pour le climat» [1, 2], lancée en 2014 en Suisse romande, qui compte maintenant une demidouzaine de groupes régionaux et plus de 500 membres, s'efforce d'ouvrir un tel dialogue à propos du dérèglement climatique.

\section{Agir ensemble}

L'association a pu le faire le 29 novembre 2018 sous le titre "Agir ensemble pour le climat». Avec l'aimable collaboration de l'Université de Lausanne, en particulier de son Centre interdisciplinaire de la durabilité placé sous l'égide du Vice-recteur Benoît Frund. Avec deux orateurs de haut vol: Martine Rebetez, la climatologue connue, et Jacques Dubochet, nobélisé il y a un an - et membre de la première heure des GPclimat! Le public a répondu à l'invitation, plus de 400 personnes dans un grand auditoire comble (certains des jeunes présents assis par terre pour laisser la place aux seniors!).

Les exposés se sont révélés complémentaires, en décrivant la situation et des perspectives sombres au vu de développements exponentiels. M. Rebetez a montré comment, sur le Plateau comme en montagne, la période d'hiver s'est raccourcie (de près de dix jours chaque décennie dans le passé récent), et comment le nombre de jours où la température dépasse 30 degrés 
augmente sérieusement. J. Dubochet: «Nous sommes très bons à créer du savoir, nous sommes mauvais s'agissant de l'utiliser pour le bien de chacun» - formule illustrant ses engagements à la fois académiques et sociaux.

\section{Jacques Dubochet: «C'est possible parce qu'il le faut!»}

Une dimension de la soirée était la participation d'une dizaine de groupes de jeunes, issus des Hautes écoles et au-delà, qui de diverses manières s'engagent pour des modes de vie plus durables et respectueux, notamment dans l'utilisation des ressources (non renouvelables, fossiles). Par des posters et en interrogeant les conférenciers, ils avaient l'occasion de présenter leurs démarches. Les questions ont porté sur les aspects politiques du combat pour le climat et le rôle de l'économie, parmi d'autres. Comment les citoyens, la so-

\section{Une dimension de la soirée était la participa- tion d'une dizaine de groupes de jeunes.}

ciété civile, peuvent-ils faire bouger les lignes? L’idée d'un recours à la force est refusée mais pas celle d'une désobéissance civile non violente. Il a été relevé que le

\section{Grands-parents pour le climat: pourquoi et comment?}

Nous, aînés, admettons que notre génération est à l'origine d'une société de consommation polluante, destructrice, avide de profit rapide. C'est ainsi que I'histoire nous jugera - en dépit des efforts que nous aurons tenté de déployer.

Nous avons, sans nous en rendre compte, mis en marche la destruction de notre milieu de vie (pour couronner le tout, nous avons l'«indécence» de vivre vieux et de devenir plus nombreux...). Cette terre, dont nous sommes locataires et non propriétaires, pourrait devenir hostile à la vie. Les dégâts sont accablants, chaque jour davantage. En fait, I'humanité et ses civilisations sont probablement plus menacées encore que la nature.

Fort-e-s de ces constatations, nous sommes aussi de plus en plus nombreux à prendre conscience des perspectives dramatiques que nous allons laisser à nos petits-enfants - sans avoir jusqu'ici su comment y remédier. Il y a à l'évidence nécessité d'un changement radical d'attitudes et de comportements individuels et collectifs, notamment modes de consommation.

Nos petits-enfants, aux premières loges, ne sont pas dupes, on observe heureusement chez eux une sensibilisation et une volonté d'agir qui s'affirment. Avec eux, nous voulons susciter un mouvement de fond dans la société, avec l'espoir que cela devienne une déferlante. Nous voulons mettre en œuvre des actions pratiques, notamment au plan local. De plus, de concert avec les jeunes générations, nous alertons les autorités, dans notre pays et au niveau international avec des associations-sœurs, pour que des mesures adéquates soient prises à large échelle, dans le sens des décisions de la COP 21 de 2015 à Paris.

Dans ces démarches, nous œuvrons avec les mouvements menant le même combat, en particulier sous le label Alliance climatique. Nous entendons user de tous les moyens à disposition pour donner à tous et à chacun la possibilité d'agir, en utilisant les canaux politiques de notre démocratie. De manière à redonner confiance à nos concitoyens, en valorisant les avantages $d^{\prime}$ une transition vers un monde plus sobre tout en promouvant une nouvelle et indispensable solidarité. pouvoir des consommateurs est important - en étant attentifs à la qualité des aliments ou équipements, à la manière dont ils sont produits, au coût de leur transport. Quelqu'un a dit: "Je suis ce que je mange.»

Martine Rebetez: «Le futur est forcément sans carbone.» Semble peu attrayant!... Pourtant, a-t-elle dit, bien que des modifications radicales du style de vie soient requises, un monde sans carbone peut être plus agréable à vivre que celui d'aujourd'hui. Certains ont appelé de leurs vœux un Mai 68 climatique!

Ce qu'on sentait dans l'air, c'est un bouillonnement, une impression que quelque chose est en train de se passer.

Jacques Dubochet sur la possibilité de stopper la dérive actuelle: "C'est possible parce qu'il le faut!» Dans la foulée, il dit joindre le pessimisme du réalisme à l'optimisme de la volonté - et de la nécessité.

Ce qu'on sentait dans l'air, c'est un bouillonnement, une impression que quelque chose est en train de se passer. Anne Mahrer, ancienne conseillère nationale genevoise, co-présidente des "Aînées pour la protection du climat» qui ont entrepris une démarche judiciaire pour contraindre le Conseil fédéral à faire plus en la matière, a parlé des vagues qui doivent devenir une déferlante.

En fin de débat, on a été au-delà des aspects scientifiques et politiques: des intervenants ont souhaité que nous entamions dans la joie les changements nécessaires, avec la sagesse d'être à l'écoute de la nature. Bonne idée sans doute.

De nos jours, les Hautes écoles savent qu'elles doivent entretenir des liens avec la société civile dans l'étude et la gestion des défis du temps. L'hospitalité offerte par l'UNIL à une simple association est un excellent signe à cet égard. Le Vice-recteur Frund a noté qu'«il n'y a pas y de diplôme d'expert en transformation" [de la société]. De tels développements sont de nature à encourager. Mais les réticences, atermoiements voire blocages, le climato-scepticisme en un mot, sont loin de n'être plus qu'une donnée historique. Pour finir, ce mot d'un participant: Une hirondelle ne fait pas le printemps, on le sait bien, mais c'était une belle hirondelle!

\section{Références}

1 Martin J. Grands-parents pour le climat. Bulletin Med Suisses / Schweiz Ärzteztg. 2014;95(45):1714. Publié en allemand sous le titre "Grosseltern für das Klima» - même réf.

2 www.gpclimat.ch

\section{Crédit photo}

(c) Photojogtom | Dreamstime.com 